\title{
Type of economic evaluations
}

\author{
Damitha Asanga Gunawardane \\ ${ }^{1}$ Office of the Regional Director of Health Services, Nuwara Eliya, Sri Lanka \\ *Correspondence: damithagunawardane@gmail.com \\ DOI: https://doi.org/10.4038/jccpsl.v25i3.8215 (iD https://orcid.org/0000-0001-8844-296X
}

Received on: 8 August 2019

Accepted on: 25 August 2019

\section{Introduction}

Economic evaluation attempts to identify ways in which limited resources can be utilized efficiently. Efficiency in health care sector requires priority to be given for those interventions, which provide the greatest benefits per unit cost. Economic evaluations involve the identification and valuation, and then the comparison of costs (inputs) and benefits (outcomes) of two or more alternative interventions. Economic evaluation is now an accepted method for the appraisal of healthcare programmes. Although it is used widely in developed countries, it is only just beginning to achieve popularity in the low- and middle-income countries (1).

\section{Types of economic evaluations}

Full economic evaluation is a study, where there are two or more alternatives and both costs and benefits are examined. Questions about the value for money can only be addressed through full economic evaluations (2). Although economic evaluations approach costs in a common format, they differ in the way they approach benefits. The types of economic evaluation are listed in Table 1.

Table 1. Types of economic evaluation

\begin{tabular}{ll}
\hline Type & Sub type \\
\hline Partial economic evaluation & Cost analysis \\
\hline Full economic evaluation & Cost minimisation analysis (CMA) \\
& Cost benefit analysis (CBA) \\
& Cost effectiveness analysis (CEA) \\
& Cost utility analysis (CUA)
\end{tabular}

Costs refer to the value of opportunities foregone because of not employing resources elsewhere. Benefits are determined by the consequences of a health intervention on people's health status. The different ways of measuring outcomes result in a trade-off between the potential scope for use and the practicality of various evaluation methods (3). An algorithm to identify the type of economic evaluation based on their characteristics is given in Figure 1. 


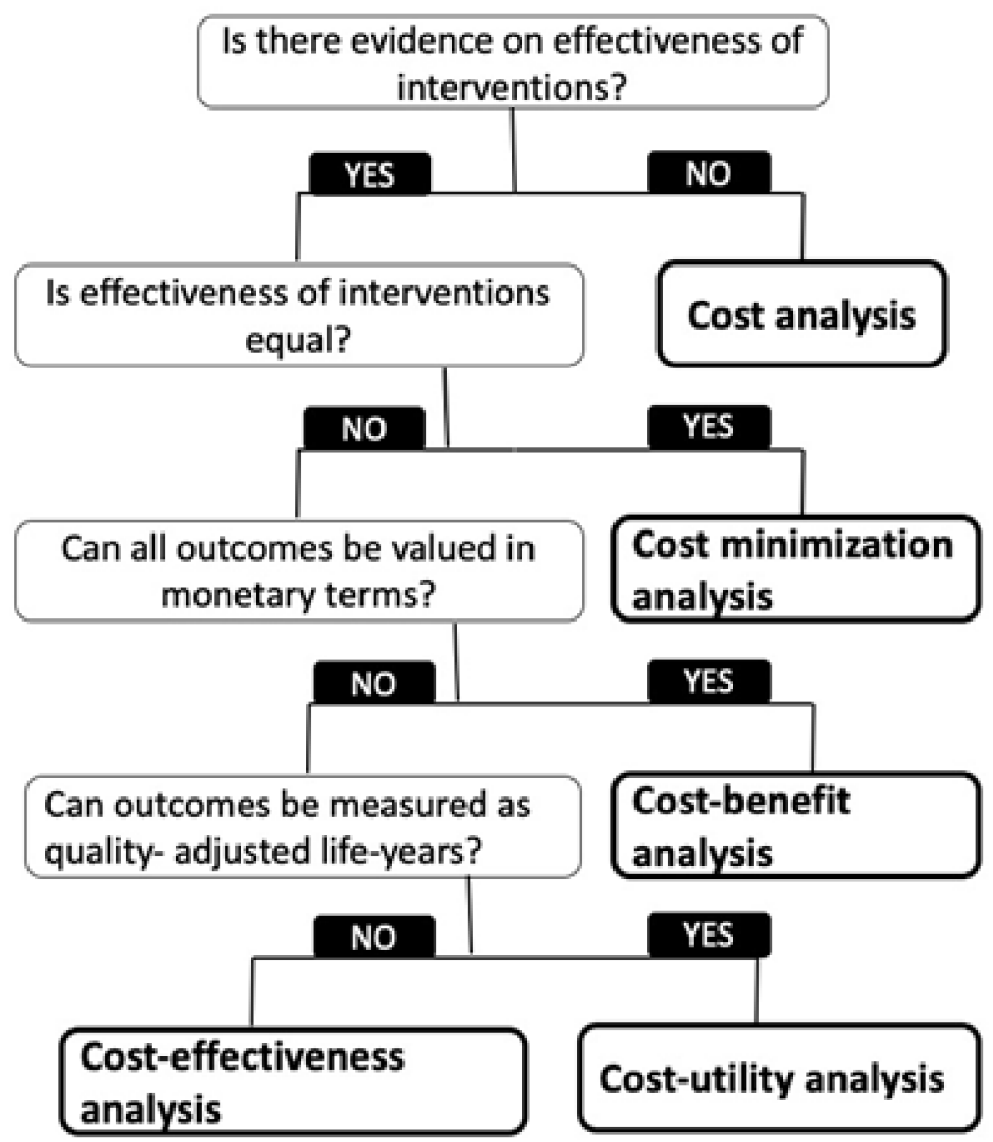

Figure 1. Algorithm to identify the type of economic evaluation based on their characteristics

Source: Gray A. Economic evaluation in Dawes, et al. (eds). Evidence based practice: a primer for health care professionals, 2001

\section{Cost analysis}

The most basic type of economic evaluation is cost analysis, which is a partial economic evaluation because it assesses only at the costs of the intervention and provides no information on the health outcome of interest. A cost analysis can be used in a situation where the effectiveness of an intervention is not yet known.

\section{Cost minimization analysis (CMA)}

This type of analysis is used when the outcomes of two interventions being compared are identical, and it is important that the outcomes of the alternative interventions are proven to be the same (4). The aim is to find the lowest cost approach and the unit of measurement is the cost per intervention. It would then be possible to calculate the costing for both methods and to select the lowest. The advantage of cost minimization analysis is that the measurement and valuing are reduced simply to examining resources utilized (Inputs). For example, if two vaccines had equivalent levels of effectiveness against a given disease, cost minimization analysis would identify which of the two vaccines would be least costly.

\section{Cost benefit analysis (CBA)}

If the outcome of two health programmes differs, then a common denominator must be established to allow comparisons of the outcome. The cost benefit analysis aims to measure the costs and the consequences in terms of monetary values (e.g. LKR, USD). CBA differs from other types of economic evaluations, in which costs and benefits of healthcare are expressed in the same units. 


\section{Cost effectiveness analysis (CEA)}

This method is used when the interventions may have differential success in the outcome, but a common outcome (e.g. blood pressure reduction). The aim of this type of analysis is to find the most efficient treatment option in terms of cost per unit effect (e.g. cost per life years saved). The disadvantage of the cost effectiveness approach is that it cannot be used to compare interventions which have several different clinical outcomes. It was this limitation, which leads to the development of cost utility analysis (CUA). There are a number of similarities between CEA and CUA and the two terms are sometimes used synonymously, even though they are not the same.

\section{Cost utility analysis (CUA)}

Cost utility analysis values outcomes using measures of utility that reflect people's preferences. The outcomes are then expressed in terms of measures such as quality- (QALYs) or disability-adjusted life years (DALYs) (5). Utility refers to the value of a particular health state or an improvement in that health state. Utility values lie between 0 and 1 , where 0 is equivalent to death and 1 is equivalent to perfect health. It is also the ideal method when interventions affect both morbidity and mortality or when interventions have a wide range of different outcomes. CUA has an advantage over CEA as it attempts to combine more than one outcome measure.

\section{Technical efficiency versus allocative efficiency}

Economic evaluation attempts to identify ways in which scarce resources can be utilized efficiently. Efficiency has two principal components in this context (5). First, there is technical (or operational) efficiency, which concentrates on maximizing the achievement of a given objective within a given budget (cheapest way to achieve a given objective). Second, there is allocative efficiency, which is focused on choosing the optimal mix of interventions for a given level of expenditure to achieve maximize health gains. This definition of efficiency allows comparisons to be made among different health care interventions with different objectives, (e.g. malaria versus TB) in order to address how a ministry of health's budget should best be distributed between programmes. Although interventions may have different outcomes of interest, all these must be converted into comparable units to discuss the allocative efficiency.

Therefore, CUA can be used to assess allocative efficiency within a given sector (e.g. health sector). However, as economic evaluation using CUA can still only compare programmes within a given sector, it can therefore only address a part of the allocative efficiency. In theory, CBA has the biggest capacity to address allocative efficiency, because the monetization of outcomes enables inter-sectoral comparisons, i.e. it can address how a government budget should be distributed between different ministries. But in practice, it is difficult to give a monetary value to health outcomes in comparison to most of the other sector outcomes. Therefore, only CBA (and CUA within the health sector) can be used to assess allocative efficiency, which can be assessed using any of the different types of economic evaluation (Table 2).

Table 2. Type of efficiency addressed through different types of economic evaluation

\begin{tabular}{lccc}
\hline Type & Outcomes & Technical efficiency? & Allocative efficiency? \\
\hline CMA & Not applicable & Yes & No \\
CBA & $\$$ & Yes & Yes \\
CEA & Natural Units & Yes & No \\
CUA & QALYs, DALYs & Yes & Yes, with health care \\
\hline
\end{tabular}

$\mathrm{CMA}=$ cost minimization analysis; $\mathrm{CBA}=$ cost benefit analysis; $\mathrm{CEA}=$ cost effectiveness analysis; $\mathrm{CUA}=$ cost utility analysis; QALYs=quality adjusted life years; DALYs=disability adjusted life years

Source: WHO. Guide for Standardization of Economic Evaluations of Immunization Programmes. Geneva: World Health Organization, 2008 


\section{References}

1. Neumann PJ, Greenberg D, Olchanski NV, Stone PW, Rosen AB. Growth and quality of the costutility literature, 1976-2001. Value in Health 2005; 8(1): 3-9.

2. Drummond MF, Sculpher MJ, Claxton K, Stoddart GL, Torrance GW. Methods for the economic evaluation of health care programmes. Oxford University Press, 2015.
3. McCluskey A. Evidence based practice: a primer for health care professionals. Australian Occupational Therapy Journal 2001; 48(4): 203-204.

4. Cunningham S. Economics: Economic evaluation of healthcare - is it important to us? British Dental Journal 2000; 188(5): 250.

5. WHO. WHO Guide for Standardization of Economic Evaluations of Immunization Programmes. Geneva: World Health Organization, 2008. 